\title{
PSEUDO HARMONIC MORPHISMS
}

\author{
E. LOUBEAU
}

\begin{abstract}
We study a geometrical condition (PHWC) which is weaker than horizontal weak conformality. In particular, we show that harmonic maps satisfying this condition, which will be called pseudo harmonic morphisms, include harmonic morphisms and can be described as pulling back certain germs to certain other germs. Finally, we construct a canonical f-structure associated to every map satisfying (PHWC) and find conditions on this fstructure to ensure the harmonicity of the map.
\end{abstract}

\section{INTRODUCTION}

Harmonic morphisms between Riemannian manifolds are maps which pull back germs of harmonic functions to germs of harmonic functions. They can be characterised as horizontally weakly conformal harmonic maps and the interplay between the analytical condition (harmonicity) and the geometrical one (horizontal weak conformality) is a rich source of properties.

In this paper we shall be interested in generalising the geometrical condition of horizontal weak conformality for maps into a Hermitian manifold to the condition of Pseudo Horizontal Weak Conformality

$$
\left[d \phi \circ(d \phi)^{*}, J\right]=0 .
$$

Harmonic maps satisfying (11) shall be called Pseudo Harmonic Morphisms. The origin of Condition (11) lies in the study of the stability of harmonic maps. In particular, Burns, Burstall, de Bartolomeis and Rawnsley showed in BBdBR89 that any stable harmonic map into an irreducible Hermitian symmetric space of compact type satisfies (1) and so, with our terminology, is a pseudo harmonic morphism. This result was then used by Chen Che93 to show that any stable harmonic map from a compact Riemannian manifold to the 2 -sphere $\mathbb{S}^{2}$ with its standard metric is a harmonic morphism.

We first investigate the geometrical implications of (11) and explain how it generalises horizontal weak conformality. Then we show its independence of the complex structure on the target.

We then give a characterisation of pseudo harmonic morphisms similar to that of harmonic morphisms, as maps which pull back germs of pluriharmonic functions to germs of harmonic functions.

Finally, to any map satisfying (11) we associate a generalised almost complex structure (f-structure) and find conditions on this structure which force the map to be harmonic.

The author would like to wholeheartedly thank J. C. Wood for numerous discussions and his unfailing guidance.

1991 Mathematics Subject Classification. Primary:58E20 Secondary:53C15.

Key words and phrases. harmonic maps, harmonic morphisms, $f$-structures. 


\section{A Special Class of Harmonic Maps}

Let $(M, g)$ be a Riemannian manifold of real dimension $m,(N, J, h)$ a Hermitian manifold of complex dimension $n$ and $\phi$ a smooth map between them.

After complexifying the tangent bundle of $N$, we identify the tangent bundles of $M$ and $N$ with their duals, $T^{*} M$ and $T^{*} N$, in order to construct the map

$$
d \phi \circ(d \phi)^{*}: T^{\mathbb{C}} N \rightarrow T^{\mathbb{C}} N .
$$

Definition 1. We say that the map $\phi:(M, g) \rightarrow(N, J, h)$ is Pseudo Horizontally Weakly Conformal if

$$
\left[d \phi \circ(d \phi)^{*}, J\right]=0 .
$$

Lemma 1. Condition $(P H W C)$ is equivalent to the condition that $d \phi \circ(d \phi)^{*}$ map the holomorphic tangent bundle $T^{(1,0)} N$ of $N$ onto itself.

Proof. Let $v \in T^{(1,0)} N$, then, since $d \phi \circ(d \phi)^{*}$ is a complex linear map, Condition (PHWC) holds if and only if

$$
i\left(d \phi \circ(d \phi)^{*}\right)(v)=J\left(d \phi \circ(d \phi)^{*}\right)(v) \quad \forall v \in T^{(1,0)} N,
$$

i.e. $\left(d \phi \circ(d \phi)^{*}\right)(v) \in T^{(1,0)} N \quad \forall v \in T^{(1,0)} N$.

Lemma 2. Set $V_{x}=d \phi_{x}^{*}\left(T^{(1,0) *}{ }_{\phi(x)} N\right)$ for $x \in M$. Then Condition $(P H W C)$ is equivalent to $V_{x}$ being isotropic $\forall x \in M$.

Proof. Let $v, w \in T^{(1,0) *}{ }_{\phi(x)} N$, then

$$
g_{x}\left(d \phi_{x}^{*}(v), d \phi_{x}^{*}(w)\right)=h_{\phi(x)}\left(v, d \phi_{x} \circ d \phi_{x}^{*}(w)\right),
$$

so that

$$
g_{x}\left(d \phi_{x}^{*}(v), d \phi_{x}^{*}(w)\right)=0 \quad \text { for all } v, w \in T^{(1,0) *}{ }_{\phi(x)} N
$$

if and only if $d \phi \circ d \phi_{x}^{*}$ maps $T^{(1,0) *} N$ to itself i.e. if and only if $\phi$ satisfies Condition (PHWC).

Lemma 3. If we equip the manifold $(M, g)$ with the local real coordinates $\left(x^{i}\right)_{i=1, \ldots, m}$ and the manifold $(N, J, h)$ with the local complex coordinates $\left(z^{\alpha}\right)_{\alpha=1, \ldots, n}$ then Condition $(P H W C)$ can be expressed as:

$$
\sum_{i, j=1}^{m} g^{i j} \frac{\partial \phi^{\alpha}}{\partial x^{i}} \frac{\partial \phi^{\beta}}{\partial x^{j}}=0 \quad \forall \alpha, \beta=1, \ldots, n .
$$

Proof. We know that Condition (PHWC) is equivalent to $d \phi_{x}^{*}\left(T_{x}^{(1,0) *} N\right)$ being isotropic and this condition reads

$$
g^{*}\left((d \phi)^{*}\left(d z^{\alpha}\right),(d \phi)^{*}\left(d z^{\beta}\right)\right)=0 \quad \forall \alpha, \beta=1, \ldots, n,
$$

where $g^{*}$ is the metric dual to $g$. But the left-hand side of the last equation is equal to:

$$
\begin{aligned}
g^{*}\left(\frac{\partial \phi^{\alpha}}{\partial x^{i}} d x^{i}, \frac{\partial \phi^{\beta}}{\partial x^{j}} d x^{j}\right) & = \\
\sum_{i, j=1}^{m} \frac{\partial \phi^{\alpha}}{\partial x^{i}} \frac{\partial \phi^{\beta}}{\partial x^{j}} g^{*}\left(d x^{i}, d x^{j}\right) & =\sum_{i, j=1}^{m} g^{i j} \frac{\partial \phi^{\alpha}}{\partial x^{i}} \frac{\partial \phi^{\beta}}{\partial x^{j}}
\end{aligned}
$$


establishing the Lemma.

Remark 1. Let $(M, g)$ be a Riemannian manifold and $(N, J, h)$ a Hermitian manifold. A map $\phi:(M, g) \rightarrow(N, J, h)$ is said to be horizontally weakly conformal if for each $p \in M$ either $d \phi_{p}=0$ or $d \phi_{p}$ is conformal and surjective on the orthogonal complement of the kernel.. The conformal factor is then called the dilation. If $\phi$ is horizontally weakly conformal then, at all points, $d \phi \circ(d \phi)^{*}=\lambda^{2} I d$ (where $\lambda$ is the dilation of $\phi$ and $I d$ is the identity map on $\left.T^{\mathbb{C}} N\right)$. Therefore any horizontally weakly conformal map satisfies Condition (PHWC), and, if $\operatorname{dim}_{\mathbb{C}} N=1$, the two notions coincide. In particular any harmonic morphism satisfies Condition (PHWC). In local coordinates this phenomenon can be explained as follow. A map is horizontally weakly conformal if and only if it satisfies

$$
g^{i j} \frac{\partial \phi^{A}}{\partial x^{i}} \frac{\partial \phi^{B}}{\partial x^{j}}=\lambda^{2} h^{A B} \quad \forall A, B=1, \overline{1}, \ldots, n, \bar{n} .
$$

If the metric $h$ is Hermitian then $h^{A B}=0$ except for $A=\alpha, B=\bar{\beta}$ and $A=\bar{\alpha}$, $B=\beta$.

If we decompose (2) into its $(1,1),(2,0)$ and $(0,2)$ parts then Condition (PHWC) is clearly the (2,0)-part of (2). Furthermore Condition (PHWC) is independent of the Hermitian metric on $N$.

Definition 2. By a local map $\psi: N \rightarrow P$, we shall mean a map defined on an open subset of $N$.

Proposition 1. Let $(M, g)$ be a Riemannian manifold (with local coordinates $\left(x^{i}\right)$ ) and $N, P$ Hermitian manifolds (with respective systems of local coordinates $\left(w^{\alpha}\right)$ and $\left.\left(u^{a}\right)\right)$. Then the map $\phi: M \rightarrow N$ satisfies Condition (PHWC) if and only if $\psi \circ \phi$ satisfies Condition (PHWC) for all local \pm holomorphic maps $\psi: N \rightarrow P$.

Proof. For any local holomorphic map $\psi$,

$$
g^{i j} \frac{\partial(\psi \circ \phi)^{a}}{\partial x^{i}} \frac{\partial(\psi \circ \phi)^{b}}{\partial x^{j}}=\frac{\partial \psi^{a}}{\partial w^{\alpha}} \frac{\partial \psi^{b}}{\partial w^{\beta}} g^{i j} \frac{\partial \phi^{\alpha}}{\partial x^{i}} \frac{\partial \phi^{\beta}}{\partial x^{j}},
$$

with a similar formula for an antiholomorphic map. This clearly shows the "if" part, the converse being shown by choosing particular holomorphic maps.

We can use Condition (PHWC) to define a class of maps which includes the notion of harmonic morphism:

Definition 3. A harmonic map which is pseudo horizontally weakly conformal shall be called a Pseudo Harmonic Morphism.

Proposition 2. Let $\phi:(M, g) \rightarrow(N, J, h)$ be a smooth map from a Riemannian manifold to a Kähler manifold.

Then $\phi$ pulls back local complex-valued \pm holomorphic functions on $N$ to local harmonic functions on $M$ if and only if $\phi$ is a pseudo harmonic morphism. In fact, in this case, $\phi$ pulls back local \pm holomorphic functions to local harmonic morphisms. 
Proof. All we need to do is to apply the chain rule for harmonic maps and use holomorphic functions as test functions:

Suppose that $\phi$ is a harmonic map and let $f: N \rightarrow \mathbb{C}$ be a local holomorphic function; then, with the usual notations:

$$
\begin{aligned}
\tau(f \circ \phi) & =d f(\tau(\phi))+\operatorname{trace} \nabla d f(d \phi, d \phi) \\
& =\operatorname{trace} \nabla d f(d \phi, d \phi) .
\end{aligned}
$$

As $(N, J, h)$ is Kähler, in complex normal coordinates this can be written at a point as:

$$
\begin{aligned}
g^{i j} \frac{\partial^{2} f}{\partial z^{A} \partial z^{B}} \frac{\partial \phi^{A}}{\partial x^{i}} \frac{\partial \phi^{B}}{\partial x^{j}} & = \\
g^{i j} \frac{\partial^{2} f}{\partial z^{\alpha} \partial z^{\beta}} \frac{\partial \phi^{\alpha}}{\partial x^{i}} \frac{\partial \phi^{\beta}}{\partial x^{j}} & =\quad \text { as } f \text { is holomorphic } \\
& =0 \quad \text { if } \phi \text { satisfies Condition (PHWC). }
\end{aligned}
$$

Thus $f \circ \phi$ is harmonic. Similarly for $f$ anti-holomorphic.

Conversely, if $\phi$ pulls back local \pm holomorphic functions on $N$ to harmonic functions on $M$ then

$$
\frac{\partial f}{\partial z^{\gamma}} \tau^{\gamma}(\phi)+g^{i j} \frac{\partial^{2} f}{\partial z^{\alpha} \partial z^{\beta}} \frac{\partial \phi^{\alpha}}{\partial x^{i}} \frac{\partial \phi^{\beta}}{\partial x^{j}}=0
$$

for any local holomorphic function $f$ on $N$. Choosing particular holomorphic functions shows that $\phi$ is harmonic and satisfies Condition (PHWC).

Let $f$ be holomorphic. Then by Proposition 1, we know that $f \circ \phi$ satisfies Condition (PHWC) and because of Remark 1 this is equivalent to horizontal weak conformality so that $f \circ \phi$ is a harmonic morphism.

Remark 2. In the case that $(N, J, h)$ is only Hermitian the proof fails as the expression for trace $\nabla d f(d \phi, d \phi)$ in local coordinates contains terms of the type

$$
g^{i j} \frac{\partial \phi^{\alpha}}{\partial x^{i}} \frac{\partial \phi^{\bar{\beta}}}{\partial x^{j}}{ }^{M} \Gamma_{\alpha \bar{\beta}}^{\gamma} \frac{\partial f}{\partial z^{\gamma}}
$$

which do not, in general, vanish.

After dealing with the case of holomorphic functions we turn our attention to the case of holomorphic maps.

Proposition 3. Let $(M, g)$ be a Riemannian manifold. Let $(N, h)$ and $(P, k)$ be two Kähler manifolds. Then the map $\phi:(M, g) \rightarrow(N, h)$ is a pseudo harmonic morphism if and only if $\psi \circ \phi:(M, g) \rightarrow(P, k)$ is a local pseudo harmonic morphism for all local \pm holomorphic maps $\psi:(N, h) \rightarrow(P, k)$..

Proof. Given $p \in M$ and $\psi: N \rightarrow P$, equip the manifold $P$ with the complex normal local coordinates $\left(w^{m}\right)_{m=1, \ldots, p}$ centred on $\psi(p)$ and $N$ with the complex normal local coordinates $\left(z^{\alpha}\right)_{\alpha=1, \ldots, n}$ centred on $p$.

Let $\phi: M \rightarrow N$ be a pseudo harmonic morphism and $\psi: N \rightarrow P$ a local holomorphic map, then the map $\psi \circ \phi$ satisfies Condition (PHWC) because of Proposition 1 . We now show that $\psi \circ \phi$ is harmonic:

$$
\begin{aligned}
\tau^{m}(\psi \circ \phi) & =d \psi^{m}(\tau(\phi))+\operatorname{trace}(\nabla d \psi)^{m}(d \phi, d \phi) \\
& =g^{i j} \frac{\partial^{2} \psi^{m}}{\partial z^{\alpha} \partial z^{\beta}} \frac{\partial \phi^{\alpha}}{\partial x^{i}} \frac{\partial \phi^{\beta}}{\partial x^{j}} \quad \text { since } \psi \text { is holomorphic } \\
& =0
\end{aligned}
$$


so $\psi \circ \phi$ is harmonic.

Conversely, if $\psi \circ \phi$ is a pseudo harmonic morphism for any local \pm holomorphic map $\psi$ then, again because of Proposition $\mathbb{1}, \phi$ satisfies Condition (PHWC).

Further, as $\psi \circ \phi$ is harmonic, we have

$$
\tau^{m}(\psi \circ \phi)=d \psi^{m}(\tau(\phi))+\operatorname{trace} \nabla d \psi(d \phi, d \phi)=0
$$

As $\psi$ is \pm holomorphic and $\phi$ satisfies Condition (PHWC) the second term is zero (cf. (4) and, by taking particular holomorphic maps, we see that $\phi$ is harmonic.

Remark 3. The "if" part of Proposition 2 is a special case of the "only if" part of Proposition 3 .

Definition 4. A smooth map from a complex manifold $N^{n}$ to a Riemannian manifold $P^{r}$ satisfying:

$$
\nabla^{(0,1)} d^{\prime} \phi=0
$$

is called a pluriharmonic map (Uda88). Here

$$
\left(\nabla^{(0,1)} d^{\prime} \phi\right)(\bar{Z}, W)={ }^{\phi} \nabla_{\bar{Z}}\left(d^{\prime} \phi(W)\right)-d^{\prime} \phi\left(\bar{\partial}_{\bar{Z}} W\right) \quad \forall Z, W \in T^{(1,0)} N^{n},
$$

where $\bar{\partial}$ is the $\bar{\partial}$-operator of the holomorphic bundle $T^{(1,0)} N^{n}$. A map is a pluriharmonic map if and only if its restriction to any holomorphic curve is harmonic (see OV90, Prop. 1.1]).

We remark that:

$$
\nabla^{(0,1)} d^{\prime} \phi=0 \Leftrightarrow \nabla^{(1,0)} d^{\prime \prime} \phi=0 .
$$

The equation for pluriharmonicity of a map is

$$
\begin{aligned}
& \frac{\partial^{2} \phi^{A}}{\partial z^{\alpha} \partial z^{\bar{\beta}}}+{ }^{X} \Gamma_{J L}^{A} \frac{\partial \phi^{J}}{\partial z^{\alpha}} \frac{\partial \phi^{L}}{\partial z^{\bar{\beta}}}=0 \\
& \forall A \in\{1, \ldots, r\} \text { and } \forall \alpha, \beta \in\{1, \ldots, n\} .
\end{aligned}
$$

Remark 4. Just like "being harmonic and satisfying Condition (PHWC)" is independent of any choice of metric on $N$ (see Proposition 3 with $(P, k)=(N, h)$ ), pluriharmonic maps are harmonic with respect to any Kähler structure on the manifold $N$.

Proposition 4. Let $\phi$ be a smooth map from a Riemannian manifold $(M, g)$ to a Kähler manifold $(N, h)$. Then $\phi$ pulls back local pluriharmonic functions on $N$ to local harmonic functions on $M$ if and only if $\phi$ is a pseudo harmonic morphism.

Proof. As a special case of Definition 4, a pluriharmonic function on a complex manifold $N$ is a map $f: N \rightarrow \mathbb{R}$ which satisfies

$$
\nabla^{(0,1)} d^{\prime} f=0
$$

In complex normal local coordinates this condition is (cf. OU90, p. 631])

$$
\frac{\partial^{2} f}{\partial z^{\alpha} \partial z^{\bar{\beta}}}=0 \quad \forall \alpha, \beta=1, \ldots, n .
$$


All we need to do is to apply the chain rule for harmonic maps:

Suppose that $\phi$ is a pseudo harmonic morphism and let $f: N \rightarrow \mathbb{R}$ be a local pluriharmonic function. Then, in normal local coordinates:

$$
\begin{aligned}
\tau(f \circ \phi) & =g^{i j} \frac{\partial^{2} f}{\partial z^{A} \partial z^{B}} \frac{\partial \phi^{A}}{\partial x^{i}} \frac{\partial \phi^{B}}{\partial x^{j}} \\
& =g^{i j} \frac{\partial^{2} f}{\partial z^{\alpha} \partial z^{\beta}} \frac{\partial \phi^{\alpha}}{\partial x^{i}} \frac{\partial \phi^{\beta}}{\partial x^{j}}+g^{i j} \frac{\partial^{2} f}{\partial z^{\bar{\alpha}} \partial z^{\bar{\beta}}} \frac{\partial \phi^{\bar{\alpha}}}{\partial x^{i}} \frac{\partial \phi^{\bar{\beta}}}{\partial x^{j}} \quad \text { as } f \text { is pluriharmonic } \\
= & 0 \quad \text { as } \phi \text { satisfies Condition (PHWC). }
\end{aligned}
$$

To prove the converse, first observe that a complex-valued function is pluriharmonic if and only if its real and imaginary parts are real-valued pluriharmonic functions, and that \pm holomorphic functions on $N$ are pluriharmonic. Therefore by taking the real (or imaginary) part of a local \pm holomorphic function on $N$ with prescribed first derivatives at a point $p \in N$, we can produce local real-valued pluriharmonic functions on $N$ with prescribed derivatives at the point $p$.

If $\phi$ pulls back local pluriharmonic functions on $N$ to harmonic functions on $M$ then

$$
\frac{\partial f}{\partial z^{\gamma}} \tau^{\gamma}(\phi)+g^{i j} \frac{\partial^{2} f}{\partial z^{\alpha} \partial z^{\beta}} \frac{\partial \phi^{\alpha}}{\partial x^{i}} \frac{\partial \phi^{\beta}}{\partial x^{j}}=0
$$

for any local pluriharmonic function $f$ on $N$.

We then choose pluriharmonic functions with prescribed first derivatives at a point, by the process described above, and proceed as in Proposition 2 .

Similarly,

Corollary 1. Let $N$ and $P$ be Kähler manifolds. Then a smooth map $\phi: M \rightarrow N$ pulls back local pluriharmonic maps from $N$ to $P$ to harmonic maps on $M$ if and only if it is a pseudo harmonic morphism.

We close this section with examples which show that Condition (PHWC) and horizontal weak conformality are not equivalent.

Example 1. Let $\phi: \mathbb{R}^{2} \rightarrow \mathbb{C}^{3}$ be defined by:

$$
\phi\left(x_{1}, x_{2}\right)=\left(\phi^{1}\left(x_{1}, x_{2}\right), \phi^{2}\left(x_{1}, x_{2}\right), \phi^{3}\left(x_{1}, x_{2}\right)\right)
$$

with

$$
\phi^{1}\left(x_{1}, x_{2}\right)=\phi^{2}\left(x_{1}, x_{2}\right)=\phi^{3}\left(x_{1}, x_{2}\right)=x_{1}+i x_{2} .
$$

It is easy to see that $\phi$ is harmonic and that it cannot be horizontally weakly conformal because it is not constant and the dimension of the target is greater than the dimension of the domain.

However

$$
\sum_{i=1,2} \frac{\partial \phi^{\alpha}}{\partial x_{i}} \frac{\partial \phi^{\beta}}{\partial x_{i}}=1^{2}+i^{2}=0, \quad \alpha, \beta=1,2,3 .
$$

So $\phi$ is harmonic and satisfies Condition (PHWC) but is not horizontally weakly conformal (it is, in fact, an immersion).

Example 2. Let $\phi: \mathbb{R}^{4} \rightarrow \mathbb{C}^{2}$ be defined by:

$$
\phi\left(x_{1}, x_{2}, x_{3}, x_{4}\right)=\left(i\left(x_{1}+x_{2}\right)+x_{3}+x_{4}, i\left(x_{1}+x_{2}\right)+x_{3}+x_{4}\right) .
$$


It is easy to see that

$$
(\operatorname{ker} d \phi)^{\perp}=\left\{\left(X_{1}, X_{2}, X_{3}, X_{4}\right) \mid X_{1}=X_{2}, X_{3}=X_{4}\right\} .
$$

If $X, Y \in(\operatorname{ker} d \phi)^{\perp}$ then

$$
\langle X, Y\rangle=2\left(X_{1} Y_{1}+X_{3} Y_{3}\right)
$$

while

$$
\langle d \phi(X), d \phi(Y)\rangle=8\left(X_{1} Y_{1}+X_{3} Y_{3}+i\left(X_{1} Y_{3}-X_{3} Y_{1}\right)\right)
$$

so that $\phi$ cannot be horizontally weakly conformal.

However $\phi$ is harmonic since linear and it is easy to verify that $\sum_{i=1}^{4}\left(\frac{\partial \phi^{\alpha}}{\partial x_{i}}\right)^{2}=0$, i.e. $\phi$ is a pseudo harmonic morphism.

\section{F-Structures And (PHWC) MAPs}

Definition 5. An f-structure on a Riemannian manifold $(M, g)$ is a (smooth) skewsymmetric section $F$ of $\operatorname{End}(T M)$ such that:

$$
F^{3}+F=0 \text {. }
$$

The definition implies that $F$ has three possible eigenvalues on the complexification of $T M:+i,-i$ and 0 . The corresponding eigenspaces $T^{+} M, T^{-} M$ and $T^{0} M$ are orthogonal with respect to the Hermitian metric $h(X, Y)=g(X, \bar{Y})$ on $T^{\mathbb{C}} M$. An almost complex structure on a manifold is an f-structure with trivial 0-eigenspace.

f-Structures were first considered by Yano [Yan63 (cf. IY64 as well as KY84, Chap. VII]). They include almost complex structures and almost contact structures.

Proposition 5. Raw85, Prop. 2.2] Let $(M, g)$ be a Riemannian manifold. There is a bijection between the f-structures $F$ of rank $2 k$ on $T M$ and the $g$-isotropic subbundles $T^{+} M$ of $T^{\mathbb{C}} M$ of rank $k$, given by $T^{+} M=\operatorname{ker}(F-i)$.

Definition 6. IY64 An f-structure $F$ of rank $2 m$ is said to be integrable if there exists a system of local coordinates adapted to $F$, i.e. in which $F$ has the constant components

$$
F=\left(\begin{array}{ccc}
0 & -\mathrm{Id}_{m} & 0 \\
\operatorname{Id}_{m} & 0 & 0 \\
0 & 0 & 0
\end{array}\right),
$$

where $\operatorname{Id}_{m}$ is the $m \times m$ identity matrix.

An f-structure $F$ is said to be parallel if

$$
(\nabla F)(X, Y)=0 \quad \forall X, Y \in T^{\mathbb{C}} M .
$$

As in the case of complex structures, parallelism implies integrability.

Theorem 1. IY64 Let $F$ be an f-structure. Define the Nijenhuis tensor $N(X, Y)$ of $F$ by

$$
N(X, Y)=[F X, F Y]-F[F X, Y]-F[X, F Y]+F^{2}[X, Y]
$$

Then the f-structure $F$ is integrable if and only if

$$
N(X, Y)=0
$$

for any two vector fields $X$ and $Y$. 
Definition 7. A map $\phi$ between two Riemannian manifolds $(M, g)$ and $(N, h)$, each carrying an f-structure $F^{M}$ and $F^{N}$, shall be called f-holomorphic if $d \phi$ intertwines the f-structures, i.e.

$$
d \phi \circ F^{M}=F^{N} \circ d \phi .
$$

Remark 5. 1. This definition implies that

$$
(d \phi)\left(T^{+} M\right) \subseteq T^{+} N,(d \phi)\left(T^{-} M\right) \subseteq T^{-} N,(d \phi)\left(T^{0} M\right) \subseteq T^{0} N .
$$

2. This choice of terminology differs slightly from Rawnsley's in Raw85 where he prefers to call such maps "morphisms of f-manifolds", reserving the term "f-holomorphic" for the cases where the domain carries an almost complex structure.

An application of f-structures to the theory of harmonic maps was given by Rawnsley in the following generalisation of a result of Lichnerowicz:

Proposition 6. Raw85 Let $(M, g, J)$ be a cosymplectic manifold and let $(N, h, F)$ be a Riemannian manifold with an f-structure which satisfies:

$$
\nabla_{X} C^{\infty}\left(T^{+} N\right) \subseteq C^{\infty}\left(T^{+} N\right) \quad \forall X \in C^{\infty}\left(T^{-} N\right) .
$$

Then every f-holomorphic map $\phi: M \rightarrow N$ is harmonic.

Definition 8. We shall say that a complex vector field $v \in C\left(T^{\mathbb{C}} M\right)$ is of type + (respectively -, 0) if $v \in C\left(T^{+} M\right)$ (respectively $v \in C\left(T^{-} M\right), v \in C\left(T^{0} M\right)$ ). Similarly for complex 1-forms $\theta \in C\left(T^{\mathbb{C} *} M\right)$.

We can associate a canonical f-structure on $T^{\mathrm{C}^{*}} M$ to each map satisfying Condition (PHWC):

Proposition 7. Let $\phi:(M, g) \rightarrow(N, h, J)$ be a smooth map from a Riemannian manifold to a Hermitian manifold.

If $\phi$ satisfies Condition (PHWC) then $(d \phi)^{*}\left(T^{(1,0) *} N\right)$ is an isotropic bundle and therefore defines an f-structure on $T^{\mathrm{c}^{*}} M$.

Definition 9. Let $(M, g)$ be a Riemannian manifold and $(N, h, J)$ a Hermitian manifold.

Let $\phi:(M, g) \rightarrow(N, h, J)$ be a smooth map satisfying Condition (PHWC). We shall call the f-structure defined by Proposition 6 , the associated $f$-structure and denote it by $F^{\phi}$.

Remark 6. Let $(M, g),(N, h, J)$ and $\phi$ be as in Definition 9. Then $\phi:\left(M, F^{\phi}, g\right) \rightarrow$ $(N, h, J)$ is f-holomorphic.

Indeed

$$
\begin{aligned}
& (d \phi)^{*}\left(T^{(1,0) *} N\right) \subseteq T^{*+} M \\
& \Leftrightarrow\left\{\begin{array}{l}
g^{*}\left((d \phi)^{*}\left(T^{(1,0) *} N\right), T^{*+} M\right)=0 \\
g^{*}\left((d \phi)^{*}\left(T^{(1,0) *} N\right), T^{* 0} M\right)=0
\end{array}\right. \\
& \Leftrightarrow\left\{\begin{array}{l}
(d \phi)\left(T^{+} M\right) \subseteq T^{(1,0)} N \\
(d \phi)\left(T^{0} M\right) \subseteq T^{(0,1)} N
\end{array}\right.
\end{aligned}
$$

but as $(d \phi)\left(T^{0} M\right)$ is real, the last equation implies

$$
(d \phi)\left(T^{0} M\right)=0 .
$$


Viewing $J$ as an f-structure, $(d \phi)^{*}\left(T^{(1,0) *} N\right) \subseteq T^{*+} M$ (and its complex conjugate) is equivalent to

$$
\begin{aligned}
& (d \phi)\left(T^{+} M\right) \subseteq T^{+} N=T^{(1,0)} N, \\
& (d \phi)\left(T^{-} M\right) \subseteq T^{-} N=T^{(0,1)} N, \\
& (d \phi)\left(T^{0} M\right) \subseteq T^{0} N=\{0\}
\end{aligned}
$$

i.e.

$$
d \phi \circ F^{\phi}=J \circ d \phi
$$

which means that $\phi$ is f-holomorphic.

Let $\phi:(M, g) \rightarrow(N, h)$ be a smooth map from a Riemannian manifold to a Hermitian manifold. Consider $(d \phi)^{*}:\left(T^{*} M, g^{*}\right) \rightarrow\left(T^{*} N, h^{*}\right)$ where $g^{*}$ and $h^{*}$ are the metrics dual to $g$ and $h$.

Then $(d \phi)^{*}$ is a smooth section of the bundle $\left(\phi^{-1} T^{*} N\right)^{*} \otimes T^{*} M$ which can be identified with the bundle $T^{*} M \otimes \phi^{-1} T N$. Let $\nabla$ denote the connection on the bundle $T^{*} M \otimes \phi^{-1} T N$ induced from the Levi-Civita connection on $M$ and $N$ then

$$
\nabla(d \phi)^{*} \in C^{\infty}\left(T^{*} M \otimes T^{*} M \otimes \phi^{-1} T N\right) .
$$

Let $X \in T M$ and $Y \in \phi^{-1} T^{*} N$. Then

$$
\begin{aligned}
\left(\nabla(d \phi)^{*}\right)(X, Y) & =\left(\nabla_{X}(d \phi)^{*}\right)(Y) \\
& =\nabla_{X}^{T^{*} M}\left((d \phi)^{*}(Y)\right)-(d \phi)^{*}\left(\nabla_{d \phi(X)}^{T^{*} N} Y\right),
\end{aligned}
$$

which is in $T^{*} M$.

If we denote by $\left(x^{i}\right)_{i=1, \ldots, m}$ a system of local coordinates on $M$ in the neighbourhood of the point $x$ and by $\left(z^{\alpha}\right)_{\alpha=1, \ldots, n}$ a system of local complex coordinates on $N$ around the point $\phi(x)$, then a simple computation shows:

\section{Lemma 4.}

$$
\sum_{i, j=1}^{m} g^{i j}\left(\nabla_{\frac{\partial}{\partial x^{i}}}(d \phi)^{*}\left(d z^{\alpha}\right)\right)\left(\frac{\partial}{\partial x^{j}}\right)=\tau^{\alpha}(\phi),
$$

where $\tau(\phi)$ is the tension field.

Proof. This follows from the calculation:

$$
\begin{aligned}
\nabla_{\frac{\partial}{\partial x^{i}}}(d \phi)^{*}\left(d z^{\alpha}\right) & =\nabla_{\frac{\partial}{\partial x^{i}}}^{T^{*} M}(d \phi)^{*}\left(d z^{\alpha}\right)-(d \phi)^{*}\left(\nabla_{d \phi\left(\frac{\partial}{\partial x^{i}}\right)}^{T^{*} N} d z^{\alpha}\right) \\
= & \frac{\partial}{\partial x^{i}}\left(\frac{\partial \phi^{\alpha}}{\partial x^{j}}\right) d x^{j}+\frac{\partial \phi^{\alpha}}{\partial x^{j}} \nabla_{\frac{\partial}{\partial x^{i}}}^{T^{*} M} d x^{j}-\frac{\partial \phi^{B}}{\partial x^{i}}(d \phi)^{*}\left(-{ }^{N} \Gamma_{B C}^{\alpha} d z^{C}\right) \\
= & {\left[\frac{\partial^{2} \phi^{\alpha}}{\partial x^{i} \partial x^{j}}-{ }^{M} \Gamma_{i j}^{k} \frac{\partial \phi^{\alpha}}{\partial x^{k}}+{ }^{N} \Gamma_{B C}^{\alpha} \frac{\partial \phi^{B}}{\partial x^{i}} \frac{\partial \phi^{C}}{\partial x^{j}}\right] d x^{j} }
\end{aligned}
$$

Theorem 2. Let $(M, g)$ be a Riemannian manifold and $(N, h, J)$ a Kähler manifold.

Let $\phi:(M, g) \rightarrow(N, h, J)$ be a non-constant smooth map satisfying Condition (PHWC). If the f-structure $F^{\phi}$ associated to $\phi$ is parallel then $\phi$ is harmonic. 
Proof. Using local coordinates $\left(x^{i}\right)_{i=1, \ldots, m}$ on $M$ adapted to the f-structure $F^{\phi}$, the tension field of $\phi$ can be written:

$$
\tau^{\alpha}(\phi)=\sum_{i, j=1}^{m} g^{i j}\left[\nabla_{\frac{\partial}{\partial x^{i}}}^{T^{*} M}(d \phi)^{*}\left(d z^{\alpha}\right)-(d \phi)^{*}\left(\nabla_{d \phi\left(\frac{\partial}{\partial x^{i}}\right.}^{T^{*}} d z^{\alpha}\right)\right]\left(\frac{\partial}{\partial x^{j}}\right) .
$$

As the tension field $\tau(\phi)$ is real, we shall only consider $\tau^{\alpha}(\phi), \alpha \in\{1, \ldots, n\}$.

Lemma 5. Let $(M, g),(N, h, J)$ and $\phi$ be as in Theorem 2 .

$$
\sum_{i, j=1}^{m} g^{i j}(d \phi)^{*}\left(\nabla_{d \phi\left(\frac{\partial}{\partial x^{i}}\right)}^{T^{*} N} d z^{\alpha}\right)\left(\frac{\partial}{\partial x^{j}}\right)=0 .
$$

Proof. We only have to consider three cases:

1) $\frac{\partial}{\partial x^{i}} \in T^{+} M$ and $\frac{\partial}{\partial x^{3}} \in T^{-} M$,

2) $\frac{\partial}{\partial x^{i}} \in T^{-} M$ and $\frac{\partial}{\partial x^{j}} \in T^{+} M$,

3) $\frac{\partial}{\partial x^{i}}, \frac{\partial}{\partial x^{j}} \in T^{0} M$.

1) When $\frac{\partial}{\partial x^{i}} \in T^{+} M$ then $d \phi\left(\frac{\partial}{\partial x^{i}}\right) \in T^{(1,0)} N$ and, since $N$ is Kähler,

$$
\nabla_{d \phi\left(\frac{\partial}{\partial x^{i}}\right)}^{T^{*} N} d z^{\alpha} \in T^{(1,0) * N}
$$

Together with Remark 6, this proves that

$$
(d \phi)^{*}\left(\nabla_{d \phi\left(\frac{\partial}{\partial x^{2}}\right)}^{T^{*} N} d z^{\alpha}\right) \in T^{*+} M,
$$

but as $\frac{\partial}{\partial x^{j}} \in T^{-} M$,

$$
(d \phi)^{*}\left(\nabla_{d \phi\left(\frac{\partial}{\partial x^{2}}\right)}^{T^{*} N} d z^{\alpha}\right)\left(\frac{\partial}{\partial x^{j}}\right)=0 .
$$

2) If $\frac{\partial}{\partial x^{i}} \in T^{-} M$ then $d \phi\left(\frac{\partial}{\partial x^{i}}\right) \in T^{(0,1)} N$ and, since $N$ is Kähler,

$$
\nabla_{d \phi\left(\frac{\partial}{\partial x^{i}}\right)}^{T^{*} N} d z^{\alpha}=0
$$

3) For $\frac{\partial}{\partial x^{i}} \in T^{0} M$, because $\phi$ is f-holomorphic, the vector $d \phi\left(\frac{\partial}{\partial x^{i}}\right)$ vanishes.

On the other hand, the condition that $F^{\phi}$ be parallel is equivalent to (cf. Raw85, Lemma 2.3]):

$$
\nabla_{X}^{T M} C^{\infty}\left(T^{+} M\right) \subseteq C^{\infty}\left(T^{+} M\right) \quad \forall X \in T M
$$

but, by considering the Christoffel symbols, it is easy to see that:

$$
\begin{aligned}
& \nabla_{T^{-} M}^{T M} C^{\infty}\left(T^{+} M\right) \subseteq C^{\infty}\left(T^{+} M\right) \Rightarrow\left\{\begin{array}{l}
\nabla_{T^{+} M}^{T^{*} M} C^{\infty}\left(T^{*+} M\right) \subseteq C^{\infty}\left(T^{*+} M+T^{* 0} M\right) \\
\nabla_{T^{*}-M}^{T^{*} M} C^{\infty}\left(T^{*+} M\right) \subseteq C^{\infty}\left(T^{*-} M+T^{* 0} M\right)
\end{array}\right. \\
& \nabla_{T^{0} M}^{T M} C^{\infty}\left(T^{0} M\right) \subseteq C^{\infty}\left(T^{0} M\right)
\end{aligned}
$$

Therefore:

$$
\nabla_{\frac{\partial}{\partial x^{i}}}^{T^{*} M}(d \phi)^{*}\left(d z^{\alpha}\right) \in \begin{cases}T^{*+} M+T^{* 0} M & \text { when } \frac{\partial}{\partial x^{i}} \in T^{+} M, \\ T^{*-} M+T^{* 0} M & \text { when } \frac{\partial}{\partial x^{i}} \in T^{-} M, \\ T^{*+} M+T^{*-} M & \text { when } \frac{\partial}{\partial x^{i}} \in T^{0} M\end{cases}
$$


Since $g^{i j}=0$ when $\frac{\partial}{\partial x^{i}}$ and $\frac{\partial}{\partial x^{j}}$ are both of the same type + or - , or when one is of type 0 and the other is of type + or - , we have that

$$
\tau^{\alpha}(\phi)=\sum_{i, j=1}^{m} g^{i j}\left[\nabla_{\frac{\partial}{\partial x^{i}}}^{T^{*} M}(d \phi)^{*}\left(d z^{\alpha}\right)\right]\left(\frac{\partial}{\partial x^{j}}\right)=0 .
$$

Remark 7. The condition on $F^{\phi}$ in Theorem 2 is a strong one. It is the analogue of being Kähler for an almost Hermitian manifold.

We can weaken the condition on the associated f-structure if we introduce a condition on the fundamental 2 -form $\omega$.

Definition 10. KY84 Let $\left(M^{m}, g\right)$ be a Riemannian manifold with an f-structure $F$. We define the fundamental 2-form of $\left(M^{m}, g, F\right)$ to be,

$$
\omega(X, Y)=g(X, F Y) \quad \forall X, Y \in T^{\mathbb{C}} M .
$$

In a frame $\left(\theta_{i}\right)_{i=1, \ldots, m}$ adapted to the f-structure $F$ :

$$
\omega=\frac{1}{2} \sum_{\substack{i<j=1 \\ i, j \in(+)}}^{m} g\left(\theta_{i}, \theta_{\bar{\jmath}}\right) \Theta^{i} \wedge \Theta^{\bar{\jmath}}
$$

where $i \in(+)$ means that $\theta_{i} \in T^{+} M,\left\{\Theta^{i}\right\}$ is the dual frame of $\left\{\theta_{i}\right\}$ and, for $i \in(+), \Theta^{\bar{\imath}}=\overline{\Theta^{i}} \in T^{*-} M$.

Theorem 3. Let $(M, g)$ be a Riemannian manifold and $(N, h, J)$ a Kähler manifold.

Let $\phi:(M, g) \rightarrow(N, h, J)$ be a non-constant smooth map satisfying Condition (PHWC). Suppose that the associated f-structure $F^{\phi}$ is integrable, and

$$
\nabla_{T^{0} M}\left(T^{*+} M\right) \subseteq T^{*+} M+T^{*-} M,
$$

and the fundamental 2-form $\omega$ is such that:

$$
d \omega^{(1,2)}=0
$$

where $d \omega^{(1,2)}=0$ means that $d \omega(u, v, w)=0$ whenever $u, v$ are of the same type and $w$ of a different type.

Then $\phi$ is a harmonic map.

Proof. Let $\left(x^{j}\right),\left(z^{\alpha}\right)$ be adapted local coordinates on $M$ and $N$. Recall that the tension field of $\phi$ can be written as:

$$
\tau^{\alpha}(\phi)=\sum_{i, j=1}^{m} g^{i j}\left[\nabla_{\frac{\partial}{\partial x^{i}}}^{T^{*} M}(d \phi)^{*}\left(d z^{\alpha}\right)-(d \phi)^{*}\left(\nabla_{d \phi\left(\frac{\partial}{\partial x^{i}}\right)}^{T^{*} N} d z^{\alpha}\right)\right]\left(\frac{\partial}{\partial x^{j}}\right)
$$

and that (Lemma 5):

It is clear that Condition (7) implies

$$
(d \phi)^{*}\left(\nabla_{d \phi\left(\frac{\partial}{\partial x^{i}}\right)}^{T^{*} N} d z^{\alpha}\right)=0 .
$$

$$
\nabla_{\frac{\partial}{\partial x^{i}}}^{T^{*} M}(d \phi)^{*}\left(d z^{\alpha}\right) \in T^{*+} M+T^{*-} M \quad \forall i \text { such that } \frac{\partial}{\partial x^{i}} \in T^{0} M .
$$


Note that Condition (7) is equivalent to

$$
\frac{\partial g_{i j}}{\partial x^{l}}=0 \quad \forall i, j \in(0), l \in(-) .
$$

It only remains to show that Condition (8) implies:

$$
\begin{aligned}
& \nabla_{\frac{\partial}{\partial x^{i}}}^{T^{*} M}(d \phi)^{*}\left(d z^{\alpha}\right) \in T^{*+} M+T^{* 0} M \quad \forall i \text { such that } \frac{\partial}{\partial x^{i}} \in T^{+} M, \\
& \nabla_{\frac{\partial}{\partial x^{i}}}^{T^{*} M}(d \phi)^{*}\left(d z^{\alpha}\right) \in T^{*-} M+T^{* 0} M \quad \forall i \text { such that } \frac{\partial}{\partial x^{i}} \in T^{-} M .
\end{aligned}
$$

Lemma 6. The fundamental 2-form $\omega$ satisfies:

$$
d \omega^{(1,2)}=0
$$

if and only if

(10) $\nabla_{v}^{T^{*} M} C^{\infty}\left(T^{*+} M+T^{*-} M\right) \in C^{\infty}\left(T^{*+} M+T^{* 0} M\right) \quad \forall v \in C^{\infty}\left(T^{+} M\right)$.

Proof. Take adapted coordinates $\left(x^{j}\right)$. Suppose, throughout the proof, that $\frac{\partial}{\partial x^{j}} \in$ $T^{+} M$.

It is easy to see that (10) is equivalent to ${ }^{M} \Gamma_{i k}^{j}=0$ whenever $d x^{i}$ has type + or and $d x^{k}$ has type - or + , different from $d x^{i}$.

We can express this condition in terms of $\omega$.

Let us first compute $d \omega$ :

$$
\begin{aligned}
d \omega= & \sum_{k=1}^{m} \sum_{\substack{i<j=1 \\
i, j \in(+)}}^{m} \frac{\partial g_{i \bar{\jmath}}}{\partial x^{k}} d x^{k} \wedge d x^{i} \wedge d x^{\bar{\jmath}} \\
= & \sum_{\substack{k<i<j=1 \\
k, i, j \in(+)}}^{m}\left(\frac{\partial g_{i \bar{\jmath}}}{\partial x^{k}}-\frac{\partial g_{k \bar{\jmath}}}{\partial x^{i}}+\frac{\partial g_{i k}}{\partial x^{\bar{\jmath}}}\right) d x^{k} \wedge d x^{i} \wedge d x^{\bar{\jmath}} \\
& +\sum_{\substack{k, i<j=1 \\
i, j \in(+), k \in(0)}}^{m} \frac{\partial g_{i \bar{\jmath}}}{\partial x^{k}} d x^{k} \wedge d x^{i} \wedge d x^{\bar{\jmath}}
\end{aligned}
$$

Notation. $d x^{k_{\alpha}} \in T^{\alpha} M$ for $\alpha=+,-, 0$.

It is easy to see that $d \omega^{(1,2)}=0$ is equivalent to

$$
\frac{\partial g_{i_{+} l_{-}}}{\partial x^{k_{-}}}=\frac{\partial g_{i_{+} k_{-}}}{\partial x^{l_{-}}} \text {. }
$$

Since

$$
{ }^{M} \Gamma_{i k}^{j}=\frac{1}{2} g^{j l_{-}}\left(\frac{\partial g_{l_{-}}}{\partial x^{i}}-\frac{\partial g_{i k}}{\partial x^{l_{-}}}+\frac{\partial g_{i l_{-}}}{\partial x^{k}}\right),
$$

we first observe that, for $d x^{i} \in T^{-} M$ and $d x^{k} \in T^{0} M$,

$$
{ }^{M} \Gamma_{i k}^{j}=0
$$

and that $d \omega^{(1,2)}=0$ implies

in both cases

$$
{ }^{M} \Gamma_{i k}^{j}=0
$$

1. $d x^{i} \in T^{+} M$ and $d x^{k} \in T^{-} M$,

2. $d x^{i} \in T^{-} M$ and $d x^{k} \in T^{+} M$. 
Hence Equation (8) implies that:

$$
\begin{aligned}
& \nabla_{T^{+} M}\left(T^{*+} M\right) \subseteq T^{*+} M+T^{* 0} M, \\
& \nabla_{T^{-} M}\left(T^{*+} M\right) \subseteq T^{*-} M+T^{* 0} M .
\end{aligned}
$$

To end the proof of Theorem 3, we deduce from Lemma 6 that (9) holds. But $g^{i j}=0$ for $i$ and $j$ both of type + or - or if $i$ is of type 0 and $j$ is not. Thus

$$
\tau^{\alpha}(\phi)=\sum_{i, j=1}^{m} g^{i j}\left[\nabla_{\frac{\partial}{\partial x^{i}}}^{T^{*} M}(d \phi)^{*}\left(d z^{\alpha}\right)\right]\left(\frac{\partial}{\partial x^{j}}\right)=0
$$

and, as $\tau(\phi)$ is real,

$$
\tau(\phi) \equiv 0
$$

Corollary 2. Let $(M, g)$ carry an integrable f-structure satisfying Equation (7) and whose fundamental 2-form satisfies (8), and let $(N, J, h)$ be an Kähler manifold. Then any f-holomorphic map $\phi: M \rightarrow N$ is harmonic.

\section{REFERENCES}

[BBdBR89] D. Burns, F.E. Burstall, P. de Bartolomeis, and J. Rawnsley. Stability of harmonic maps of Kähler manifolds. J. Differential Geom., 30:579-594, 1989.

[Che93] J.Y. Chen. Stable harmonic maps into $S^{2}$. In T. Kotake, S. Nishikawa, and R. Schoen, editors, Geometry and Global Analysis, pages 431-436. Tôhoku Univ., Sendai, 1993.

[IY64] S. Ishihara and K. Yano. On integrability conditions of a structure $f$ satisfying $f^{3}+$ $f=0$. Quart. J. Math. Oxford, 15(2):217-222, 1964.

[KY84] M. Kon and K. Yano. Structures on Manifolds, volume 3 of Series in Pure Mathematics. World Scientific, 1984.

[OU90] Y. Ohnita and S. Udagawa. Stability, complex-analyticity, and constancy of pluriharmonic maps from compact Kähler manifolds. Math. Z., 205:624-644, 1990.

[OV90] Y. Ohnita and G. Valli. Pluriharmonic maps into compact Lie groups and factorization into unitons. Proc. London Math. Soc., 61:546-570, 1990.

[Raw85] J. Rawnsley. $f$-structures, $f$-twistor spaces and harmonic maps. In E. Vesentini, editor, Geometry Seminar "Luigi Bianchi" II-1984, volume 1164 of Lecture Notes in Math., pages 85-159. Springer, Berlin, Heidelberg, New York, 1985.

[Uda88] S. Udagawa. Pluriharmonic maps and minimal immersions of Kähler manifolds. $J$. London Math. Soc., 37:375-384, 1988.

[Yan63] K. Yano. On a structure defined by a tensor field of type $(1,1)$ satisfying $f^{3}+f=0$. Tensor N.S., 14:99-109, 1963.

Current address: Department of Pure Mathematics, University of Leeds, LS2 9JT Leeds, U.K.

(from 1st September 1996), Université de Bretagne Occidentale, UfR Sciences et Techniques, Departement de Mathematiques, 6, avenue Victor Le Gorgeu, BP 809, 29285 BREST CEDEX, France

E-mail address: pmt5el@amsta.leeds.ac.uk 